\title{
Angiotensin II Type 2 Receptor Gene Polymorphism in Caucasian Children With a Wide Spectrum of Congenital Anomalies of the Kidney and Urinary Tract
}

\author{
EKATERINI SIOMOU, IOANNA BOUBA, KONSTANTINOS D. KOLLIOS, FREDERICA PAPADOPOULOU, MARIA SYRROU, \\ IOANNIS GEORGIOU, AND ANTIGONI SIAMOPOULOU
}

\begin{abstract}
Department of Pediatrics [E.S., K.D.K., A.S.], Laboratory of Human Reproductive Genetics [I.B., I.G.], Department of Radiology [F.P.], University Hospital of Ioannina, 45500 Ioannina, Greece; Laboratory of General Biology [M.S.], Medical School, University of Ioannina, 45110 Ioannina, Greece
\end{abstract}

\begin{abstract}
The A-1332G transition of the angiotensin II type 2 receptor $(A T 2 R)$ gene was found to occur more often in males with ureteropelvic (UPJO) or ureterovesical junction obstruction (UVJO). However, other studies have shown controversial results. The frequency of this polymorphism was investigated in 275 Caucasian children (153 boys, 122 girls) with a wide spectrum of congenital anomalies both of upper (165) and lower (110) urinary tract system and in 200 controls (100 boys, 100 girls). Among the included malformations, renal agenesis and duplex collecting system (DCS) were studied for the first time. The frequency of the $\mathrm{G}$ allele did not differ among patients (193 of 397 total alleles, 48.6\%) and controls (146 of $300,48.7 \%$ ). No significant difference was also found in the frequency of the $G$ allele in subgroups of congenital uropathies compared with controls. When analysis was performed in males and females separately, no significant difference was found in the frequency of the $\mathrm{G}$ allele in male $(45.1 \%)$ or female $(50.8 \%)$ patients compared with male $(57.0 \%)$ or female $(44.5 \%)$ controls. Our data indicate that the $A T 2 R$ gene A-1332G transition is not associated with the development of human congenital uropathies and further investigations should be carried out to unravel their etiology. (Pediatr Res 62: 83-87, 2007)
\end{abstract}

$\mathrm{C}$ ongenital anomalies of the kidney and urinary tract (CAKUT) account for up to $30 \%$ of all anomalies diagnosed prenatally and constitute the main cause of chronic renal failure in infants and young children. Although the etiology of most of these anomalies has not been identified, experimental studies have identified several genes that are implicated in nephrogenesis and in which the derangement result in renal maldevelopment $(1,2)$.

In recent years, published data have shown that the reninangiotensin system, besides its role in maintaining blood pressure as well as fluid and electrolytes homeostasis, has an important role in kidney development (3). AT2R is abundantly expressed in fetal tissues (4) and decreases rapidly after birth (5). It has been shown to mediate programmed cell death, playing an important role in developmental biology and pathophysiology (4). The expression of the AT2R is higher and localized to the mesenchyme at the time of the ureteric bud

Received November 2, 2006; accepted February 19, 2007.

Correspondence: Ekaterini Siomou, M.D., Ph.D., Emmanouil Xanthou 58, GR-454 45 Ioannina, Greece; e-mail: eksiomou@yahoo.gr branching (3). The gene encoding AT2R is located on the X chromosome, so that normal males carry only one copy, whereas normal females carry two copies. It consists of three exons and two introns, with the entire coding region located on exon 3 (6).

Experimental studies have shown that the establishment of CAKUT is preceded by delayed apoptosis of the undifferentiated mesenchymal cells that initially occupy the metanephros and densely surround the wolffian duct and ureter (7). This abnormal apoptosis hinders the normal interaction between the ureteric bud and metanephric blastema, resulting in CAKUT (8). Moreover, At2r gene null mutant mice display CAKUT, which mimics human CAKUT (7).

Studies on the human $A T 2 R$ gene identified a polymorphic locus in a large proportion of the general population, with a nucleotide $A$ to $G$ transition at position -1332 within the lariat branch point motif of intron 1 (A-1332G polymorphism). The $\mathrm{G}$ allele seems to be associated with abnormal splicing of pre-mRNA, producing mRNA with a shorter length (exon 2 is missing) in a smaller amount than the normal allele A. This confirms the functional significance of this polymorphism. Moreover, a significant association between UPJO and this polymorphism was found in 36 Caucasian patients (7).

The majority of subsequent studies included male patients with some CAKUT $(9,10)$, whereas only one study included both males and females with various CAKUT (11). Conversely, the above studies show divergent results, from a higher frequency of the A-1332G transition in Caucasians $(9,11)$ to a similar one in Japanese (10) with various CAKUT compared with controls.

To clarify the role of $A T 2 R$ in the development of CAKUT, the distribution of the A-1332G polymorphism was examined in a large number of Caucasian children (males and females) with a wide spectrum of CAKUT because the majority of reported studies have included a relatively limited number,

Abbreviations: AT2R, angiotensin II type 2 receptor; CAKUT, congenital anomalies of the kidney and urinary tract; DCS, duplex collecting system; MCDK, multicystic dysplastic kidney; PUV, posterior urethral valves; RHD, renal hypodysplasia; ${ }^{\mathbf{9 9 m}}$ Tc-DMSA, technetium-99m dimercaptosuccinic acid; UPJO, ureteropelvic junction obstruction; UVJO, ureterovesical junction obstruction; VUR, vesicoureteral reflux 
mainly of male patients. Moreover, renal agenesis and duplex collecting system (DCS), which had not been studied previously, were included in the present study for the first time.

In the long term, an understanding of the genetic aspects of CAKUT will help to unravel the pathogenesis of these disorders and may facilitate the design of genetic screening tests for an early diagnosis (1).

\section{METHODS}

Patients. A total of 275 Caucasian children (153 boys, 122 girls) who were diagnosed as having CAKUT or followed because of known CAKUT in our Pediatrics Department from September 2001 to December 2006 were enrolled in the study. The mean age at the time of diagnosis was $2.0 \pm 2.9 \mathrm{y}$.

The clinical data included patient history, physical examination, laboratory data, and radiographic investigation, which were performed because of a history of a first urinary tract infection (167 patients: 79 males and 88 females) or prenatal hydronephrosis (108 patients: 74 males and 34 females). Imaging studies included urinary tract ultrasonography, voiding cystourethrography, and technetium-99m dimercaptosuccinic acid $\left({ }^{99 \mathrm{~m}} \mathrm{Tc}-\mathrm{DMSA}\right)$ scan of all patients. Diuretic renography and i.v. urography were performed if there was a clinical indication. All the imaging studies were performed in the Radiology Department of our hospital. Patients with syndromes or other chronic diseases were excluded from the study.

The clinical characteristics of patients are summarized in Table 1.

The control group consisted of 200 healthy Caucasian children (100 boys, 100 girls). The mean age at the time of diagnosis was $2.3 \pm 3.7 \mathrm{y}$. These children also had a history of a first urinary tract infection and were evaluated during the same period as the patients by ultrasonography, voiding cystourethrography, and DMSA in our Pediatrics and Radiology Departments, 1-2 mo after urinary tract infection. They all had normal renal function, no urological anomalies, and no chronic diseases. The controls came from the same geographic region as the patients, and there were no ethnic differences between patients and controls.

Written consent for the genetic study was obtained from parents of patients and controls. The study protocol was approved by the Ethics Committee of our hospital.

Renal agenesis was diagnosed by complete absence of the kidney on abdominal ultrasound and ${ }^{99 \mathrm{~m}} \mathrm{Tc}-\mathrm{DMSA}$ scans; renal aplasia by tiny renal tissue on ultrasound scan, without any function on DMSA scan; and multicystic dysplastic kidney (MCDK) by multiple cysts on ultrasound scan, without any function on ${ }^{99 \mathrm{~m}} \mathrm{Tc}$-DMSA. Renal hypoplasia and dysplasia can coexist, and the diagnosis can be confirmed only by renal histology. Thus, we used the term renal hypodysplasia (RHD) for kidneys with reduced renal length and regular outline, with or without hyperechogenicity of the cortex and loss of corticomedullary differentiation on ultrasonography and with persistent (for $>6 \mathrm{mo}$ ) generally reduced ${ }^{99 \mathrm{~m}} \mathrm{Tc}-\mathrm{DMSA}$ uptake. For the radiographic diagnosis of RHD to be more accurate, only the severe cases were included (split function $\leq 35 \%$ ). DCS was diagnosed by a band of

Table 1. Clinical characteristics of the patient group

\begin{tabular}{lrrrc}
\hline & \multicolumn{3}{c}{ No. of patients } & \\
\cline { 2 - 4 } CAKUT & Total & M & F & M-to-F ratio \\
\hline Upper urinary tract system & 165 & 83 & 82 & 1.0 \\
Renal maldevelopment & 116 & 66 & 50 & 1.3 \\
Renal agenesis & 28 & 16 & 12 & 1.3 \\
Renal aplasia, MCDK & 23 & 13 & 10 & 1.3 \\
RHD & 23 & 14 & 9 & 1.6 \\
RHD with VUR & 42 & 23 & 19 & 1.2 \\
DCS & 49 & 17 & 32 & 0.5 \\
Lower urinary tract system & 110 & 70 & 40 & 1.8 \\
Ureteral obstruction & 36 & 27 & 9 & 3.0 \\
UPJO & 24 & 17 & 7 & 2.4 \\
UVJO & 12 & 10 & 2 & 5.0 \\
Primary VUR & 61 & 30 & 31 & 1.0 \\
PUVs & 13 & 13 & & \\
Total & 275 & 153 & 122 & 1.3 \\
\hline
\end{tabular}

M, male; F, female; MCDK, multicystic dysplastic kidney; VUR, vesicoureteral reflux. parenchyma separating the two pelvic complexes on ultrasound scan, confirmed by i.v. urography in all cases. UPJO or UVJO was diagnosed by pelvocalyceal dilatation or both pelvicalyceal and ureteral dilatation on ultrasound scan, and findings of significant obstruction on i.v. urography and diuretic renography (12). Primary vesicoureteral reflux (VUR) was diagnosed by voiding cystourethrography and was classified as grade I-V, according to the International Reflux Classification (13). Renal scarring was defined by the presence of decreased uptake of ${ }^{99 \mathrm{~m}} \mathrm{Tc}$-DMSA, associated with loss of the contours of the kidney, or by the presence of cortical thinning with decreased volume $\geq 6$ mo after a well-documented urinary tract infection (14). Posterior urethral valves (PUVs) were diagnosed by bilateral ureterohydronephrosis, a thick-walled bladder, and dilated posterior urethra on ultrasonography and voiding cystourethrography, confirmed by cystoscopy (12).

Genetic analysis. Genomic DNA from each individual was isolated from peripheral blood leukocytes using a standard $\mathrm{NaCl}$ extraction procedure technique. The $A T 2 R$ gene A-1332G polymorphism was determined by polymerase chain reaction (PCR) amplification followed by digestion using the restriction enzyme EcoRI according to the method described by Nishimura et al. (7). All the patients and controls were successfully genotyped using the above method.

Statistical analysis. The results were analyzed with STATISTICA 6.0 for windows. The statistical methods used were the $\chi^{2}$ test and the Fisher' s exact probability test. $p$ Values $<0.05$ were considered statistically significant.

\section{RESULTS}

Patient population. Family history revealed that 20 of 275 patients (7.3\%) (all with VUR) had a positive family history of VUR as well. One girl with bilateral RHD had a father who also had bilateral RHD.

Among the patients, 204 (74\%) had unilateral CAKUT. The malformed kidney was on the right side in $89(44 \%)$ and on the left side in 115 (56\%) patients. Forty-three patients (16\%) (29 with VUR, 12 with DCS, two with RHD) had the same CAKUT bilaterally, 15 patients (5\%) had VUR in the contralateral kidney, and one patient with renal agenesis had contralateral RHD.

Among the patient with RHD, 23 had no lower urinary tract anomalies, whereas in 42, a moderate or severe VUR coexisted. Those patients had no history of urinary tract infection, but a history of prenatal hydronephrosis. In the DCS group, complete ureteral duplication was found in 26 and incomplete in 23 patients. Coexisting anomalies were VUR in 23 DCSs and ectopic ureterocele of the upper pole ureteral orifice in seven complete DCSs. The ureteroceles were accompanied by a poorly functioning upper pole in all cases and by VUR of the lower pole in five cases. In UPJO and UVJO, split function $<40 \%$ was found in five and two patients, respectively. In the VUR patients, DMSA scan showed normal findings in 55 $(90 \%)$ and renal scarring in six $(10 \%)$. Among the controls, scarring was noted in eight of $200(4 \%)$.

Genetic analysis. PCR restriction length polymorphism analysis demonstrated that the frequency of A and G alleles did not differ between Caucasian patients with CAKUT, categorized as a total or subgroups of CAKUT and controls (Table 2).

Because the $A T 2 R$ gene is located on the $\mathrm{X}$ chromosome, statistical analysis was also performed in males and females separately. There was no significant difference in the frequency of the $\mathrm{G}$ allele between male or female patients with CAKUT and male or female controls. When both sexes were categorized as subgroups of CAKUT, a lower frequency of G allele was found in males with ureteral obstruction $(p=0.03)$ and a higher in females with DCS $(p=0.02)$ compared with 
Table 2. Frequency of the AT2R gene A-1332G polymorphism in controls and patients with CAKUT

\begin{tabular}{lcc}
\hline \multicolumn{1}{c}{ Subjects } & A alleles, no. (\%) & G alleles, no. (\%) \\
\hline Controls & $154(51.3)$ & $146(48.7)$ \\
Patients & $204(51.4)$ & $193(48.6)$ \\
Renal maldevelopment & $92(55.4)$ & $74(44.6)$ \\
$\quad$ Renal agenesis & $23(57.5)$ & $17(42.5)$ \\
$\quad$ Renal aplasia, MCDK, RHD & $36(55.4)$ & $29(44.6)$ \\
RHD, associated with VUR & $33(54.1)$ & $28(45.9)$ \\
DCS & $35(43.2)$ & $46(56.8)$ \\
Ureteral obstruction & $27(60.0)$ & $18(40.0)$ \\
Primary VUR & $44(47.8)$ & $48(52.2)$ \\
PUVs & $6(46.2)$ & $7(53.8)$ \\
\hline
\end{tabular}

There was no significant difference in the frequency of each allele between controls and patients, either when they were categorized as CAKUT generally or as subgroups of CAKUT.

controls (Table 3). When the genotype distribution of the A-1332G polymorphism was studied in female patients, no significant difference was found compared with controls. Because of the limited number of several subgroups of CAKUT in females, the statistical analysis was only performed on the total of renal malformations, DCS and VUR. The unique significant difference found was the higher frequency of GG genotype in females with DCS $(p=0.03)$ compared with controls (Table 4). The values predicted by assumption of Hardy-Weinberg equilibrium for the three genotypes in females were similar to those observed in the CAKUT groups with a sufficient number of patients (total CAKUT, total renal maldevelopment, DCS, VUR) and in the control group.

When statistical analysis was performed separately in patients with urinary tract infection, no significant difference was found in the frequency of $\mathrm{G}$ allele in them (127 of 255 total alleles, 49.8\%), compared with controls (146 of 300 total alleles, 48.7\%). The same results were found when statistical analysis was performed separately in patients with prenatal hydronephrosis (66 of 142 total alleles, 46.5\%), compared with controls (146 of 300 total alleles, 48.7\%).

No significant differences were also found either in males with urinary tract infection (37 of 79, 46.8\%) or females with urinary tract infection (90 of 176, 51.1\%) compared with male (57 of $100,57.0 \%$ ) or female ( 89 of $200,44.5 \%$ ) controls. The same was noticed both in males with prenatal hydroureteronephrosis (32 of 74, 43.2\%) and females with prenatal hy-
Table 4. Genotype distribution of the AT2R A-1332G polymorphism in female controls and patients with CAKUT

\begin{tabular}{lrcc}
\hline & \multicolumn{3}{c}{ Females: genotypes, no. (\%) } \\
\cline { 2 - 4 } & AA & AG & GG \\
\hline Controls & $30(30.0)$ & $51(51.0)$ & $19(19.0)$ \\
Patients & $31(25.4)$ & $58(47.5)$ & $33(27.1)$ \\
Renal maldevelopment & $18(36.0)$ & $20(40.0)$ & $12(24.0)$ \\
DCS & $5(15.6)$ & $15(46.9)$ & $12(37.5)^{*}$ \\
Primary VUR & $6(19.4)$ & $18(58.1)$ & $7(22.5)$ \\
\hline$* p=0.03$ compared with controls. & &
\end{tabular}

droureteronephrosis (34 of $68,50.0 \%$ ) compared with male (57 of 100, 57.0\%) and female (89 of $200,44.5 \%$ ) controls.

\section{DISCUSSION}

In the present study, the distribution of the A-1332G polymorphism of the $A T 2 R$ gene was examined in a large number of Caucasian children with various CAKUT. The spectrum of CAKUT studied included renal aplasia, MCDK, RHD, RHD coexisting with VUR, ureteral obstruction, VUR, PUV, and, for the first time, renal agenesis and DCS. The association of VUR and RHD was shown by Hiraoka et al. (15) in Japanese perinatal screening studies, in which congenital small kidneys were strongly correlated with VUR.

Family history was positive for VUR in $7.3 \%$ of included patients, and only one girl had a positive family history of other CAKUT. Although most cases of CAKUT appear to be sporadic, in some of these (VUR, renal maldevelopment, UPJO, and PUV), a genetic pathogenesis is strongly suggested by a positive family history and genetic linkage studies $(1,2)$. The relatively low incidence of positive family history in the present and previous studies $(10,11)$ may be the result of the usually unilateral occurrence of CAKUT, which, being asymptomatic, may not be diagnosed throughout the lifetime.

The frequency of the $\mathrm{G}$ allele (48.7\%) among Caucasian male and female controls was similar to that among other Caucasian (46\%) (16) and Korean (55.1\%) (17) male and female controls. However, Rigoli et al. (11) reported a lower frequency $(30.4 \%)$ of the $\mathrm{G}$ allele in Italian males and females with no urological anomalies. When only males were compared, the frequency of the $\mathrm{G}$ allele was higher in our control

Table 3. Frequency of the AT2R gene A-1332G polymorphism, separately in male and female controls and patients with CAKUT

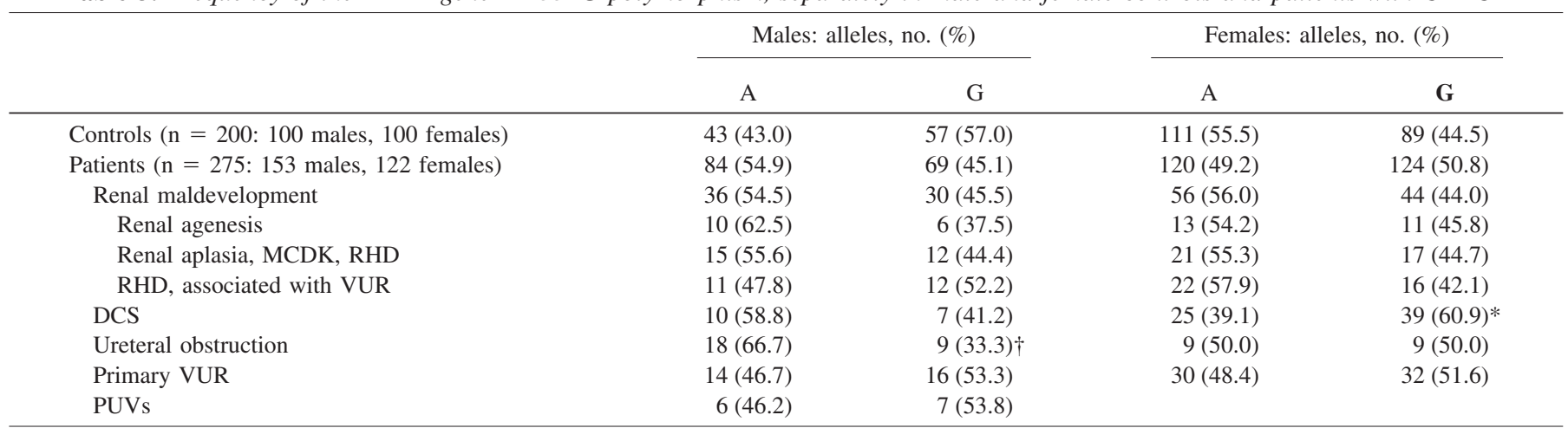

$* p=0.02$ compared with controls.

$\dagger p=0.03$ compared with controls. 
Table 5. Literature review for the frequency of the $G$ allele in patients with CAKUT and controls

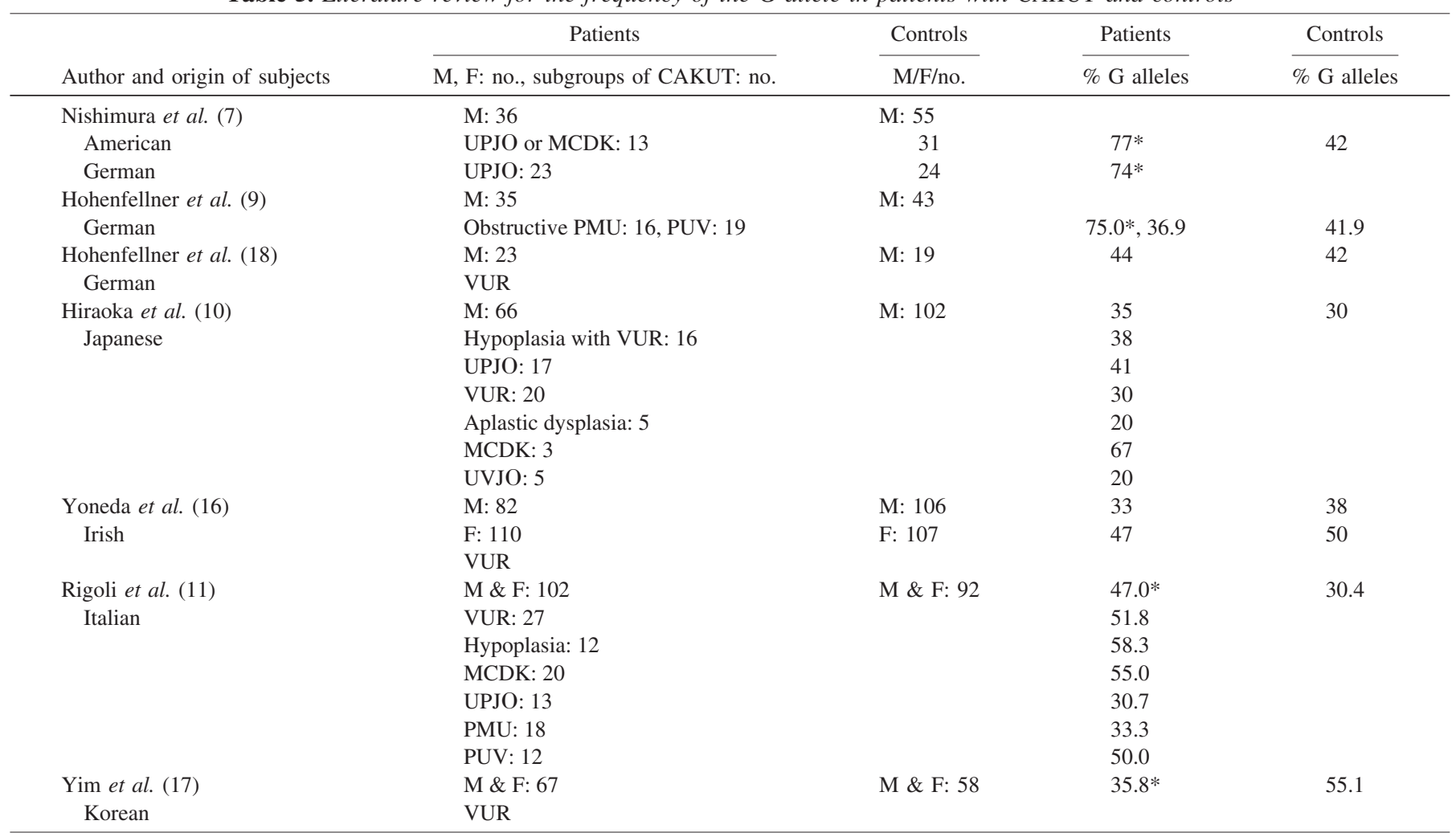

PMU, primary megaureter.

$* p<0.05$ compared with controls.

population $(57 \%)$ compared with other Caucasian (38\%$42 \%)(7,9,16,18)$ and Japanese (30\%) (10) controls. In Table 5 , previous studies considering the $\mathrm{A}-1332 \mathrm{G}$ transition in patients with CAKUT are summarized. The discrepancy of the results between Caucasian and Japanese controls could be explained by their different genetic background, but this may not be the cause for the discrepancy among Caucasians of different ethnicity. Conversely, it is notable that the control population included in all the above studies was characterized as normal using ultrasound findings. However, it is known that the sensitivity and specificity of ultrasonography are low to exclude some minor CAKUT, such as VUR (19). The control group in the present study was chosen even though they had a history of urinary tract infection 1-2 mo before their enrollment in the study because their ultrasound scan, voiding cystourethrography, and DMSA scan showed an absence of CAKUT. This was done to avoid the enrollment of children as controls, who, being asymptomatic, could not be diagnosed as having CAKUT because CAKUT usually are unilateral and asymptomatic.

No significant difference was found in the frequency of the A-G transition between Caucasian patients (both males and females) and controls when they were categorized as a total of CAKUT nor as subgroups of CAKUT. To our knowledge, the only study that included Caucasian patients with various CAKUT in both sexes was the one by Rigoli et al. (11). In that study, a significantly higher frequency of the $\mathrm{G}$ allele in Italian patients with CAKUT was found compared with controls, but not when the same patients were categorized as having sub- groups of CAKUT. Recently, Hahn et al. (20) investigated the allele frequencies of the $A T 2 R$ gene in 53 Korean patients (boys and girls) with some CAKUT and reported a significantly higher frequency of the $G$ allele in patients with the above urological anomalies compared with the general population . However, the investigators based their statistical analysis on boys as having two copies of the $A T 2 R$ gene. Because it is well documented that the $A T 2 R$ gene is located on the $\mathrm{X}$ chromosome and normal males carry only one copy and normal females two copies (6), we did not take their results into consideration.

When comparisons were made in males and females separately in the present study, again no significant difference was found in the frequency of the G allele in the total of CAKUT compared with controls. Studying the frequency in each anomaly separately, there was also no significant difference in males or females with renal maldevelopment compared with controls, nor did Hiraoka et al. (10) find any significant difference in males with hypoplasia associated with VUR. Because no previous studies have examined the frequency of the $\mathrm{G}$ allele separately in females with renal maldevelopment, direct comparisons cannot be made. No significant difference was also found in males with DCS, whereas a higher frequency of the $G$ allele and the GG genotype was found in females with DCS compared with male and female controls. Because DCS is twice as common in females (21), we can speculate that the higher frequency of the A-1332G transition in females could play a role in the development of this anomaly. However, further studies are needed to establish this 
finding. A lower frequency of $\mathrm{G}$ allele in males with ureteral obstruction was found in our population. On the contrary, previous studies showed a significantly higher frequency in Caucasian males with UPJO or UVJO $(7,9)$, whereas another study in Japanese males showed no difference (10). The discrepancy among these studies is more likely to be the result of the limited number of included patients because the genetic background, at least among Caucasians, does not differ. The present study showed no significant difference in the frequency of the A-1332G transition between patients with VUR and controls, consistent with previous studies in Caucasian and Japanese patients $(10,11,16,18)$. In contrast, Yim et al. (17) found that this transition occurred significantly less often in Korean patients (males and females) with VUR than in normal controls. Previous studies $(9,11)$, including ours, showed no significant difference in the frequency of the A-G transition in patients with PUV, which is expected because the At2 $r$ mRNA expression is absent in the urethral area (7).

Except for Japanese and Korean patients, in whom the different genetic backgrounds could explain the discrepancy in frequency of the A-1332G transition between patients and controls, the existing heterogeneity of the results among Caucasian patients cannot be explained by the genetic background. It seems more likely that this may be the result of the relatively limited number of included patients and controls. The present study comprised the largest number of patients with CAKUT for studying the $A T 2 R$ gene A- $1332 \mathrm{G}$ polymorphism compared with previously reported similar studies. Experimental studies have shown that the anatomical spectrum of CAKUT in null mutants for the $A T 2 R$ gene is impressively wide, documenting that a common gene abnormality can result in different CAKUT (7). Thus, although in the present study the number of included patients in a few subgroups of CAKUT is also limited, the large total number of patients and controls is considered sufficient enough to support our results.

In conclusion, our data indicate that the $A T 2 R$ gene A-1332G transition is not associated with the development of the CAKUT. This suggests that some other factors may play a major role in the development of human CAKUT, and further investigations should be carried out to unravel their etiology, possibly through multicenter studies.

Acknowledgments. The authors thank Katsaraki Aphrodite, statistician, for her help in the computer analysis of the data and Charilaos Kostoulas for his help in a part of the DNA extraction.

\section{REFERENCES}

1. Woolf AS 2000 A molecular and genetic view of human renal and urinary tract malformations. Kidney Int 58:500-512

2. Woolf AS, Winyard PJ 2002 Molecular mechanisms of human embryogenesis: developmental pathogenesis of renal tract malformations. Pediatr Dev Pathol 5:108 129

3. Sequeira Lopez ML, Gomez RA 2004 The role of angiotensin II in kidney embryogenesis and kidney abnormalities. Curr Opin Nephrol Hypertens 13:117-122

4. Yamada T, Horiuchi M, Dzau VJ 1996 Angiotensin II type 2 receptor mediates programmed cell death. Proc Natl Acad Sci U S A 93:156-160

5. Kakuchi J, Ichiki T, Kiyama S, Hogan BL, Fogo A, Inagami T, Ichikawa I 1995 Developmental expression of renal angiotensin II receptor genes in the mouse. Kidney Int 47:140-147

6. Koike G, Horiuchi M, Yamada T, Szpirer C, Jacob HJ, Dzau VJ 1994 Human type 2 angiotensin II receptor gene: cloned, mapped to the $\mathrm{X}$ chromosome, and its mRNA is expressed in the human lung. Biochem Biophys Res Commun 203:1842-1850

7. Nishimura H, Yerkes E, Hohenfellner K, Miyazaki Y, Ma J, Hunley TE, Yoshida H, Ichiki T, Threadgill D, Phillips JA 3rd, Hogan BM, Fogo A, Brock JW 3rd, Inagami T, Ichikawa I 1999 Role of the angiotensin type 2 receptor gene in congenital anomalies of the kidney and urinary tract, CAKUT, of mice and men. Mol Cell $3: 1-10$

8. Pope JC 4th, Brock JW 3rd, Adams MC, Stephens FD, Ichikawa I 1999 How they begin and how they end: classic and new theories for the development and deterioration of congenital anomalies of the kidney and urinary tract, CAKUT. J Am Soc Nephrol 10:2018-2028

9. Hohenfellner K, Hunley TE, Schloemer C, Brenner W, Yerkes E, Zepp F, Brock JW 3rd, Kon V 1999 Angiotensin type 2 receptor is important in the normal development of the ureter. Pediatr Nephrol 13:187-191

10. Hiraoka M, Taniguchi T, Nakai H, Kino M, Okada Y, Tanizawa A, Tsukahara H, Ohshima Y, Muramatsu I, Mayumi M 2001 No evidence for AT2R gene derangement in human urinary tract anomalies. Kidney Int 59:1244-1249

11. Rigoli L, Chimenz R, Di Bella C, Cavallaro E, Caruso R, Briuglia S, Fede C, Salpietro CD 2004 Angiotensin-converting enzyme and angiotensin type 2 receptor gene genotype distributions in Italian children with congenital uropathies. Pediatr Res 56:988-993

12. Avni FE, Hall M 2004 Diagnostic imaging. In: Avner ED, Harmon WE, Niaudet $P$ (eds) Pediatric Nephrology. Lippincott Williams \& Wilkins, Philadelphia, pp 449473

13. International Reflux Study Committee 1981 Medical versus surgical treatment of primary vesicoureteral reflux: report of the International Reflux Study Committee. Pediatrics 67:392-400

14. Hoberman A, Charron M, Hickey RW, Baskin M, Kearney DH, Wald ER 2003 Imaging studies after a first febrile urinary tract infection in young children. N Engl J Med 348:195-202

15. Hiraoka M, Hori C, Tsukahara H, Kasuga K, Ishihara Y, Sudo M 1997 Congenitally small kidneys with reflux as a common cause of nephropathy in boys. Kidney Int 52:811-816

16. Yoneda A, Cascio S, Green A, Barton D, Puri P 2002 Angiotensin II type 2 receptor gene is not responsible for familial vesicoureteral reflux. J Urol 168:1138-1141

17. Yim HE, Jung MJ, Choi BM, Bae IS, Yoo KH, Hong YS, Lee JW, Kim SK 2004 Genetic polymorphism of the renin-angiotensin system on the development of primary vesicoureteral reflux. Am J Nephrol 24:178-187

18. Hohenfellner K, Hunley TE, Yerkes E, Habermehl P, Hohenfellner R, Kon V 1999 Angiotensin II, type 2 receptor in the development of vesico-ureteric reflux. BJU Int $83: 318-322$

19. Hellstrom M, Jacobsson B 1999 Diagnosis of vesico-ureteric reflux. Acta Paediatr Suppl 88:3-12

20. Hahn H, Ku SE, Kim KS, Park YS, Yoon CH, Cheong HI 2005 Implication of genetic variations in congenital obstructive nephropathy. Pediatr Nephrol 20:15411544

21. Siomou E, Papadopoulou F, Kollios KD, Photopoulos A, Evagelidou E, Androulakakis Ph, Siamopoulou A 2006 Duplex collecting system diagnosed during the first 6 years of life after a first urinary tract infection: a study of 63 children. J Urol 175:678-681 